\title{
Estudo dos efeitos de produtos rejuvenescedores no comportamento físico, químico e reológico de ligante asfáltico envelhecido
}

\author{
Effects of rejuvenating products on the physical, chemical and rheological \\ behavior of an aged asphalt binder
}

\author{
João Paulo Souza Silva', Márcio Muniz de Farias² \\ 1Universidade Federal de Goiás, Goiás-Brasil, jpss@ufg.br \\ ${ }^{2}$ Universidade de Brasília, Distrito Federal - Brasil, muniz@unb.br
}

\section{Recebido:}

17 de outubro de 2018

Aceito para publicação:

16 de março de 2020

Publicado:

31 de agosto de 2020

Editor de área:

Kamilla Vasconcelos

\section{Palavras-chaves: \\ Ligante Asfáltico. \\ Rejuvenescedor. \\ Pavimentação.}

\section{Keywords:}

Asphalt Binder.

Rejuvenating.

Paving.

DOI:10.14295/transportes.v28i3.1874

\begin{abstract}
RESUMO
A reciclagem é um tema que vem ganhando espaço em diversas áreas da engenharia. Quando se reutiliza um material em obras civis, indiretamente há uma contribuição para a preservação da natureza. Dentro deste contexto, a reciclagem de revestimentos asfálticos é um assunto que desperta grande interesse, mas que requer estudos mais aprofundados. Assim, este trabalho tem como objetivo estudar os efeitos de produtos rejuvenescedores sobre o comportamento do ligante asfáltico envelhecido, para possível aplicação na reciclagem de revestimentos de pavimentos asfálticos. Inicialmente foi extraído o ligante residual e, em seguida, foram adicionados rejuvenescedores a base de alcatrão (RejuvaSeal) e outro a base de óleo de xisto, comercialmente conhecido como AR-5, AR-250 e AR-500, nas taxas de 15,20 e $25 \%$, a fim de verificar qual a porcentagem seria apropriada para recuperar as características originais do ligante envelhecido. Dentre as principais conclusões encontradas neste estudo, destaca-se que a mistura contendo $20 \%$ de AR-5 apresenta o melhor desempenho físico e mecânico quando comparada a uma mistura contendo ligante asfáltico novo (CAP 50-70).
\end{abstract}

\section{ABSTRACT}

Recycling is a topic that has been gaining enough space in various areas of engineering. When one reuses materials in civil construction works, there is an indirect contribution to the preservation of nature. Within this context, the recycling of asphalt layers is a subject that draws great interest but requires further study. This paper aims to study the effects of rejuvenating agents on aged asphalt binders, for their application in recycling of asphalt mixtures. The aged binder was initially extracted and then rejuvenating agents were added, i. e., a tar-based agent (RejuvaSeal) and shale-oil residues commercially designated as AR-5, AR-250 and AR-500, at percentages of 15,20 and $25 \%$ by mass, in order to determine which content would be appropriate to recover the original characteristics of the binder. Among the many findings from this study is that the mixture containing $20 \%$ of AR- 5 shows better behavior when compared to a new asphalt binder (CAP 50-70).

\section{INTRODUÇÃO}

O Brasil é um país de dimensões continentais, somando mais de 207 milhões de habitantes distribuídos irregularmente sobre cerca de 8,5 milhões de $\mathrm{km}^{2}$, (IBGE, 2017) dotado de uma malha rodoviária pavimentada relativamente pequena em relação à malha total, por vezes em mal estado de conservação, com poucas ferrovias e uma rede fluvial de baixa utilização (CNT, 2018). 
A deterioração verificada nos pavimentos brasileiros é em grande parte causada pelo excesso de carga por eixo dos veículos transportadores e pela falta de manutenção das rodovias, aliada às intempéries. Estes mecanismos causam rompimento da camada de revestimento provocando assim grande desconforto e prejuízo ao usuário.

0 meio técnico rodoviário tem se esforçado para buscar novas tecnologias e desenvolver soluções mais econômicas de restauração dos pavimentos, muitos já bastante deteriorados. Entre as novas metodologias estudadas, destaca-se a reciclagem dos revestimentos asfálticos, que além de apresentar rapidez executiva e custos competitivos, preza pelo aspecto ambiental (Silva, 2011).

A reciclagem de misturas asfálticas vem sendo executada no Brasil desde meados da década de 1980, principalmente após a introdução de máquinas fresadoras no país. Este tipo de reciclagem é uma técnica de reparação, onde ocorre o reaproveitamento total ou parcial do material fresado, também conhecido com RAP (Reclaimed Asphalt Pavement), que, traduzido para o português, seria pavimento asfáltico recuperado (Zubaran, 2014). O RAP é o velho revestimento asfáltico moído, retirado da faixa de rolamento. Esse material pode ser reutilizado em novas misturas asfálticas, pois seus componentes ainda podem apresentar propriedades satisfatórias, porém, para que isso ocorra, geralmente são adicionados outros materiais, possibilitando a construção de nova camada com características funcionais e mecânicas semelhantes ou até mesmo superiores à antiga (Silva, J., 2011; Pradyumna et al. 2013).

A reciclagem dos pavimentos é uma alternativa útil, pois reduz a utilização de agregados virgens e a quantidade de ligante asfáltico virgem necessária na produção de misturas asfálticas. O uso de material reciclado também economiza energia, reduz os custos de transporte necessários para obter agregados virgens de qualidade e preserva os recursos naturais. Entretanto, para que o material fresado seja reciclado, é necessário que se recupere essa mistura asfáltica envelhecida por meio da incorporação de aditivos químicos, chamados agentes rejuvenescedores, que têm como finalidade repor os compostos aromáticos e resinas para que as propriedades originais do ligante sejam restabelecidas.

Diante deste fato, este trabalho foi idealizado e desenvolvido na tentativa de se testar as condições de aplicação de agentes rejuvenescedores no RAP, por meio de ensaios químicos e ambientais preconizados na literatura e normas técnicas vigentes.

\section{REJUVENESCIMENTO DE MISTURAS ASFÁLTICAS}

Devido ao efeito da temperatura, oxigênio, luz solar, chuva e tempo, o ligante asfáltico tem sua consistência aumentada, promovendo o envelhecimento, promovendo uma maior rigidez da camada betuminosa. 0 concreto asfáltico ao envelhecer sofre aumento relativo no teor de asfalteno e diminuição no teor de resinas e óleos aromáticos (Bonfim e Rodrigues, 1995).

Assim, para recuperar as propriedades originais do CAP, principalmente quando se trabalha com a reutilização de misturas asfálticas envelhecidas, utiliza-se uma técnica que visa à reutilização dos agregados e do aglutinante (ligante) do revestimento envelhecido. Para tal é necessária a incorporação de aditivos químicos ao material envelhecido, os chamados agentes rejuvenescedores ou agentes de reciclagem (AR), capazes de reduzir a viscosidade e recompor as resinas e os compostos aromáticos para que as propriedades originais do ligante sejam restabelecidas (Silva, 2011).

Segundo Zaumanis et al. (2014), para misturas de alto conteúdo RAP, a aplicação de rejuvenescedores é inevitável. Nos EUA comumente é feita dosagem a partir de propriedades 
do ligante que se deseja obter, como por exemplo o grau PG, já na Alemanha, os parâmetros utilizados são o ponto de amolecimento e a penetração. No entanto, é recomendável realização de estudo caso a caso para determinação da melhor combinação RAP-rejuvenescedor.

Os rejuvenescedores à base de óleo de xisto e à base de alcatrão são os principais produtos disponíveis atualmente no mercado para esta finalidade. Um dos agentes rejuvenescedores é fornecido pela empresa brasileira de petróleo (Petrobrás). Este produto, à base de óleo de xisto, é dividido em duas categorias (a quente e a frio), e regido pelo regulamento técnico DNC 04/97 portaria ${ }^{\circ} 44$ de 29/09/1997(DNC, 1997). Os agentes a quente são denominados "Aditivos Asfálticos de Reciclagem para Misturas a Quente" (AR) e os a frio recebem o nome de "Agentes de Reciclagem Emulsionados" (ARE). Cada um deles apresenta seis tipos em função da viscosidade do ligante.

A utilização de agentes rejuvenescedores é capaz de reverter o processo de envelhecimento pelo qual o ligante passou, restaurando suas propriedades e possibilitando a reciclagem total da mistura asfáltica envelhecida (Zaumanis et al., 2014). 0 AR é utilizado na recomposição da consistência original do ligante asfáltico presente no revestimento que está sendo reciclado, pois contém compostos aromáticos que visam devolver ao asfalto a característica original de ductilidade e consistência (propriedades reológicas) perdidas pelo envelhecimento ao longo do tempo de serviço do pavimento. Os ARs são asfaltos de baixa consistência, usados em serviços de reciclagem a quente.

A seleção do tipo de AR a ser empregado no serviço de reciclagem dependerá das características de envelhecimento do ligante presente no revestimento a ser reciclado. Quanto mais envelhecido estiver o ligante asfáltico residual (medido em termos de penetração ou viscosidade) menos consistente deverá ser o AR a ser usado (Lima, 2003). A escala de consistência de óleo de xisto é composta por AR-1 (menor consistência) e AR-500 (maior consistência). Outro agente rejuvenescedor encontrado no mercado brasileiro é o produto a base de alcatrão que foi desenvolvido pela empresa americana PRI (Pavement Rejuvenation International), conhecido comercialmente pelo nome de RejuvaSeal $®$, este é comercializado no mercado nacional pela empresa SPM que se associou à empresa americana.

0 "RejuvaSeal" é um tratamento à base de alcatrão para pavimento asfáltico, proveniente de uma mistura cuidadosa de diferentes materiais e possui duas funções principais: restaurar e proteger o pavimento asfáltico envelhecido. Mah (2008) comenta que a composição do agente rejuvenescedor à base de alcatrão é basicamente Alcatrão (35-50\%), Destilado de Petróleo (3242\%) e Rejuvenescedor 15-40\%).

No Brasil uma pesquisa realizada por Silva et al. (2010), mostra que corpos de prova revestidos com RejuvaSeal ${ }^{\circledR}$ e submetidos às ações de imersão em água, gasolina, e a combinação destes, apresentaram uma menor perda de resistência à tração e de módulo de resiliência do que as amostras correspondentes sem revestimento. Os resultados dos testes e avaliações estatísticas para a combinação água+gasolina apresentou o maior potencial agressor, seguido da gasolina e por fim o efeito isolado da água.

\section{MATERIAIS E MÉTODOS}

Quatro rejuvenescedores foram utilizados na pesquisa, sendo três à base óleo de xisto e um à base de alcatrão. Os agentes rejuvenescedores à base de óleo de xisto respectivamente AR-5, AR-250 e AR-500, escolhidos de modo a representar as consistências mínima e máxima disponíveis no mercado brasileiro e um ligante com consistência intermediária. 0 rejuvenescedor à 
base de alcatrão não é um produto nacional e é conhecido comercialmente com o nome de RejuvaSeal $®$ e foi adquirido junto ao Grupo SPM, sediado em São Paulo-SP, sendo este grupo responsável pela importação e comercialização do produto no Brasil.

A obtenção do material foi realizada na Estrada Parque Indústria e Abastecimento (EPIA), na altura do Viaduto Ayrton Senna, em Brasília-DF. Este trecho estava sendo submetido ao corte com fresadora a frio do tipo Wirtgen 2100 DC apenas da camada de revestimento da pista de rolamento, originalmente formada por CBUQ, Faixa C, composto por 5\% de CAP 50-70 e 95\% de agregado micaxístico. Após a fresagem, o material foi estocado no pátio do $2^{\circ}$ Distrito Rodoviário do Departamento de Estradas de Rodagem do Distrito Federal (DER-DF).

\subsection{Extração e caracterização do ligante presente no fresado}

Foram retiradas, logo após estocagem, amostras da pilha de material fresado que, posteriormente, foram encaminhadas ao laboratório para execução de ensaios de caracterização, tais como granulometria e percentagem de betume (método Rotarex). Inicialmente, foi separado o ligante asfáltico envelhecido dos agregados utilizando processo de centrifugação (equipamento Rotarex) e destilação pelo método do Rotavaporador. Após separar o ligante dos agregados minerais e do material pulverulento, o mesmo foi levado ao destilador (Figura 1) a fim de fazer a separação entre o CAP envelhecido e o solvente (percloroetileno). 0 teor do asfalto no material fresado foi determinado conforme equação abaixo:

$$
T=\frac{M a-M d}{M a} x 100
$$

onde: Ma: $\quad$ massa da amostra antes da extração e Md = massa da amostra após a extração.
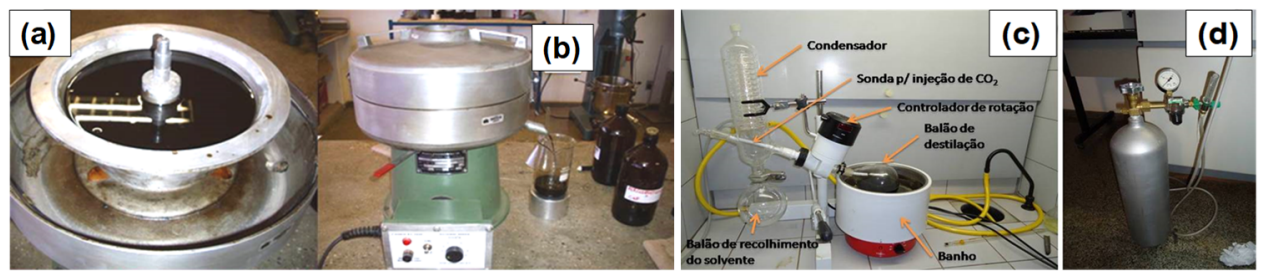

Figura 1. (a) Cuba do Rotarex com a mistura fresado-solvente ; (b) Equipamento Rotarex; (c) Equipamento Rotavaporador ; (d) Balão de CO2

Neste processo, foi utilizado o método Rotavaporador, em conformidade com a norma americana ASTM D 5404 (ASTM, 1995) (Recuperação de Asfalto da Solução usando o Equipamento Rotavaporador) salvo algumas adaptações como no caso do uso da glicerina no banho, pois quando se utiliza água como preconiza a norma, não é possível atingir a temperatura suficiente para que o solvente seja evaporado e condensado para sua posterior captação.

\subsection{Cromatografia (SARA)}

No processo de envelhecimento, tanto de curto prazo (usinagem, manuseio e aplicação) quanto de longo prazo (em serviço), ocorre normalmente uma diminuição do teor de óleos aromáticos, que se transformam em resina, que por sua vez se transformam parte em asfaltenos, resultando ao final do processo em uma pequena ou nenhuma variação do teor de saturados e resinas, diminuição do teor de aromáticos e aumento no teor de asfaltenos. Tudo isso resulta no endurecimento do ligante e respectiva rigidez da camada asfáltica. 
A cromatografia sobre coluna de sílica IATROSCAN é uma técnica que permite separar diretamente estes constituintes do ligante asfáltico em função de sua polaridade, obtendo-se assim as quatro famílias químicas SARA (saturados, aromáticos, resinas e asfaltenos), propostos por Corbett (1969). A partir do teor de cada fração SARA pode-se monitorar a evolução da composição química do ligante asfáltico envelhecido após mistura com diferentes rejuvenescedores. Também foi determinado o Ic (Índice de Instabilidade Coloidal), que segundo Gaestel (Gaestel et. al, 1971), é calculado conforme a Equação 2.

$$
I c=\frac{S+A}{R+A r}
$$

onde: $\quad \mathrm{S}$ : $\quad$ saturados, $\mathrm{A}=$ asfaltenos; $\mathrm{R}=$ resinas e $\mathrm{Ar}=$ aromáticos, todos em $\%$.

\subsection{Espectrometria de ressonância magnética nuclear - RMN}

A técnica de ressonância magnética nuclear (RMN) fundamenta-se na determinação de propriedades de uma substância por meio da correlação da energia absorvida contra a frequência em megahertz (MHz) do espectromagnético. Tornou-se um eficiente método para analisar produtos de petróleo, sendo que os espectros (comprimento de onda ou frequência) do CAP geralmente apresentam duas regiões de deslocamentos químicos distintos que correspondem às regiões de alifáticos e aromáticos, este último, mais difícil de analisar devido à grande superposição de picos.

Esta dificuldade é relatada por alguns autores como Shakirullah et. al (2006), Jennings \& Pribani (1991) e Silva \& Farias (2018), 1H e 13C dos ligantes asfálticos que se apresentam como uma mistura complexa de compostos orgânicos. Esta técnica foi utilizada para avaliação das alterações estruturais que a mistura de rejuvenescedores poderia ocasionar no ligante envelhecido que havia sido extraído do material fresado. Pretendia-se verificar se os compostos aromáticos e saturados haviam sido restabelecidos, tomando como parâmetro um CAP novo do tipo 50-70, largamente utilizado em projetos de pavimentação asfáltica na região Centro Oeste do Brasil.

Devido à indisponibilidade de equipamentos nos laboratórios da Universidade de Brasília, optou-se por realizar os ensaios, os mesmos foram terceirizados, sendo utilizado o equipamento espectrômetro de RMN marca VARIAN, modelo MR-400 e campo magnético de 9,4T (400 $\mathrm{MHz}$ em 1H).

\subsection{Caracterização dos ligantes}

Após a extração do ligante do material fresado, o CAP envelhecido naturalmente foi também caracterizado com a mesma bateria de ensaios do CAP virgem, ocorrendo o mesmo com o CAP rejuvenescido. Esses ensaios são: penetração, ponto de amolecimento, viscosidade, ductilidade, densidade, variação de massa (RTFOT), ponto de fulgor.

Os teores de incorporação dos agentes rejuvenescedores AR5, AR250, AR500 e RejuvaSeal, foram respectivamente 15\%, 20\% e 25\% em relação à porcentagem de CAP envelhecido que existia no fresado (que era de $5 \%$ em relação ao peso da mistura asfáltica), ou seja, para cada $100 \mathrm{~g}$ de CAP velho, foram incorporados 15, 20 e $25 \mathrm{~g}$ dos rejuvenescedores.

Posteriormente, o CAP virgem (CAP 50-70), CAP velho e os CAP's rejuvenescidos foram levados à estufa RTFOT nas condições especificadas nas normas, de modo a verificar sua estabilidade com relação ao envelhecimento em curto prazo. Em seguida, foram caracterizados para determinação da variação nas propriedades do material. 
Com a finalidade de determinar parâmetros viscoelásticos dos ligantes estudados nesta pesquisa (envelhecido, natural e rejuvenescido), foram realizadas medidas de módulo complexo de cisalhamento $\left(\mathrm{G}^{*}\right)$ e ângulo de fase $(\delta)$ a temperaturas variando entre 46 a $76^{\circ} \mathrm{C}(46,52,58,64$, 70 e $76^{\circ} \mathrm{C}$ ) e frequência de carregamentos de $1,59 \mathrm{~Hz}$, com uma amostra de $25 \mathrm{~mm}$ de diâmetro, no Reômetro de Cisalhamento Dinâmico (DSR), conforme preconizado pela norma AASHTO M 332 (AASHTO, 2014)

\section{RESULTADOS E ANÁLISES}

\subsection{Ensaios tradicionais (análises físicas)}

Os dados obtidos na campanha experimental são apresentados de forma resumida na Tabela 1 e Tabela 2 .

Tabela 1 - Resultados da caracterização física dos ligantes analisados

\begin{tabular}{|c|c|c|c|c|c|c|c|}
\hline \multirow[b]{2}{*}{ Ensaios } & \multirow[b]{2}{*}{ Unid. } & \multirow{2}{*}{ Norma CAP 50-70 } & \multirow{2}{*}{ CAP 50-70 } & \multirow{2}{*}{ CAP velho } & \multicolumn{3}{|c|}{ RejuvaSeal } \\
\hline & & & & & $15 \%$ & $20 \%$ & $25 \%$ \\
\hline Penetração & $0,1 \mathrm{~mm}$ & $50-70$ & 53,0 & 40,0 & 130,3 & 169,7 & 171,5 \\
\hline Pto. Amolec. & ${ }^{\circ} \mathrm{C}$ & 46,0 & 46,5 & 51,0 & 41,8 & 37,3 & 30,0 \\
\hline \multicolumn{8}{|c|}{ Viscosidade Brookfield para Ligantes } \\
\hline a $135^{\circ} \mathrm{C}$ & $\mathrm{cP}$ & 274 & 385 & 545 & 325 & 310 & 305 \\
\hline a $150^{\circ} \mathrm{C}$ & $c P$ & 112 & 165 & 256 & 273 & 240 & 168 \\
\hline a $177^{\circ} \mathrm{C}$ & $c P$ & $57-285$ & 60 & 99 & 143 & 135 & 135 \\
\hline $\mathrm{G}^{*} / \operatorname{sen} \delta(70 \circ \mathrm{C})$ & $\mathrm{kPa}$ & $>1$ & 1,0 & 1,2 & 0,4 & 0,3 & 0,3 \\
\hline IST & - & $(-1,5)$ a $(+0,7)$ & -2.0 & -1.4 & -1.0 & -2.0 & -6.1 \\
\hline \multicolumn{8}{|c|}{ Efeito do calor e do ar (RTFOT) a $163^{\circ} \mathrm{C}, 85 \mathrm{~min}$} \\
\hline$\Delta$ massa & $\%$ & $\leq 0,5$ & 0,16 & - & 4,22 & 5,19 & 7,01 \\
\hline $\mathrm{G} * / \operatorname{sen} \delta\left(70^{\circ} \mathrm{C}\right)$ & $\mathrm{kPa}$ & $>2$ & 1,5 & - & 5,1 & 6,2 & 5,7 \\
\hline$\Delta$ Pto. Amolec. & $\stackrel{\circ}{C}$ & $\leq 8$ & 1,5 & - & 15,5 & 19,0 & 26,8 \\
\hline Penetração Retida & $\%$ & $\geq 55$ & 73 & - & 14,0 & 10,0 & 10,0 \\
\hline
\end{tabular}

Tabela 2 - Resultados da caracterização física dos ligantes analisados

\begin{tabular}{|c|c|c|c|c|c|c|c|c|c|c|}
\hline \multirow{2}{*}{ Ensaios } & \multirow{2}{*}{ Unid. } & \multicolumn{3}{|c|}{ AR-500 } & \multicolumn{3}{|c|}{ AR-250 } & \multicolumn{3}{|c|}{ AR-5 } \\
\hline & & $15 \%$ & $20 \%$ & $25 \%$ & $15 \%$ & $20 \%$ & $25 \%$ & $15 \%$ & $20 \%$ & $25 \%$ \\
\hline Penetração & $0,1 \mathrm{~mm}$ & 39,8 & 43,5 & 44,3 & 44,3 & 46,0 & 49,8 & 65,6 & 68,3 & 88,5 \\
\hline Pto. Amolec. & ${ }^{\circ} \mathrm{C}$ & 49,3 & 50,0 & 49,8 & 49,0 & 47,5 & 47,8 & 47,7 & 47,2 & 46,1 \\
\hline \multicolumn{11}{|c|}{ Viscosidade Brookfield para Ligantes } \\
\hline a $135^{\circ} \mathrm{C}$ & $\mathrm{cP}$ & 535 & 500 & 490 & 478 & 455 & 428 & 335 & 285 & 250 \\
\hline a $150^{\circ} \mathrm{C}$ & $c P$ & 280 & 265 & 263 & 258 & 243 & 233 & 183 & 155 & 135 \\
\hline a $177^{\circ} \mathrm{C}$ & $\mathrm{cP}$ & 123 & 115 & 80 & 113 & 110 & 105 & 85 & 75 & 65 \\
\hline $\mathrm{G}^{*} / \operatorname{sen} \delta\left(70^{\circ} \mathrm{C}\right)$ & $\mathrm{kPa}$ & 1,3 & 1,1 & 1,1 & 1,2 & 1,0 & 1,0 & 1,1 & 1,3 & 1,1 \\
\hline IST & - & $-1,9$ & $-1,5$ & $-1,5$ & $-1,7$ & $-2,0$ & $-1,8$ & $-1,2$ & $-1,2$ & $-0,8$ \\
\hline \multicolumn{11}{|c|}{ Efeito do calor e do $\operatorname{ar}(\mathrm{RTFOT})$ a $163^{\circ} \mathrm{C}, 85 \mathrm{~min}$} \\
\hline$\Delta$ massa & $\%$ massa & 0,17 & 0,54 & 0,45 & 0,46 & 0,42 & 0,53 & 0,23 & 0,24 & 0,27 \\
\hline $\mathrm{G} * / \operatorname{sen} \delta(70 \circ \mathrm{C})$ & $\mathrm{kPa}$ & 3,0 & 2,9 & 2,9 & 2,6 & 2,3 & 2,2 & 2,3 & 2,2 & 2,2 \\
\hline$\Delta$ Pto. Amolec. & ${ }^{\circ} \mathrm{C}$ & 4,8 & 3,3 & 3,0 & 4,0 & 5,0 & 2,5 & 2,0 & 2,8 & 0,9 \\
\hline Penetração Retida & $\%$ & 54 & 49 & 49 & 45 & 46 & 49 & 78 & 83 & 69 \\
\hline
\end{tabular}

É possível observar que a adição dos rejuvenescedores favoreceu a diminuição da consistência do ligante envelhecido. Entretanto em alguns casos essa adição não foi suficiente para elevar a penetração do ligante rejuvenescido para no mínimo 50 décimos de milímetro. 0 RejuvaSeal proporcionou à mistura uma penetração muito elevada e um ponto de amolecimento muito baixo nos três teores aplicados, o que inviabiliza o uso do material nestas porcentagens, indicando que os teores testados são exagerados para esse tipo de material. 
Além disso, observa-se que todos os rejuvenescedores utilizados pela pesquisa proporcionaram aumento na penetração, mas quando submetido ao envelhecimento em curto prazo a penetração diminuiu consideravelmente, com exceção da mistura utilizando AR5 principalmente no teor de $20 \%$ após o RTFOT, indicando que para esta característica este é o material que melhor se aproxima do valor da penetração do CAP “virgem” (CAP 50-70).

O RejuvaSeal nas três porcentagens analisadas teve uma alta perda de massa, indicando um alto índice de volatilidade e uma grande capacidade de perda das frações leves, uma vez que apresentava aspecto oleoso e mais fluido que os demais rejuvenescedores estudados nesta pesquisa.

Com exceção da configuração CAPvelho+20\% AR-500, e do CAPvelho+25\% AR-250, cuja perda de massa foi de $0,54 \%$ e $0,53 \%$, os demais rejuvenescedores tiveram comportamento parecido entre si, tendo a sua variação da perda de massa da ordem de $0,23 \%$ a $0,54 \%$ o que caracteriza um bom comportamento quando comparados ao limite máximo de 0,5\% para um CAP 50-70.

\subsection{Reologia dos ligantes asfálticos}

O ensaio de cisalhamento dinâmico foi realizado para todos os ligantes analisados nesta pesquisa, ressaltando o interesse em determinação do parâmetro indicativo da deformação permanente que é governada pelo valor de $\mathrm{G}^{*} / \operatorname{sen} \delta$., para temperaturas variando entre 46 a $70^{\circ} \mathrm{C}$ e frequência de carregamento de 1,59 Hz. A Figura 2, Figura 3 e Figura 4 apresentam os resultados de $\mathrm{G}^{*} / \operatorname{sen} \delta$ obtidos.
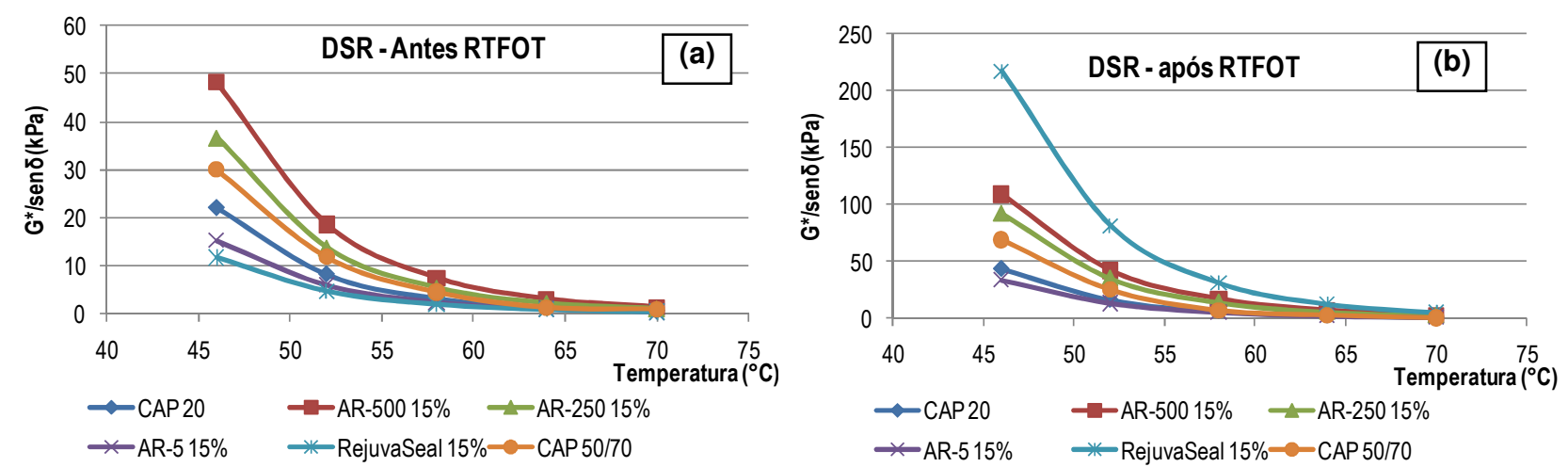

Figura 2. (a) G*/sen $\delta$ dos ligantes nos teores de adição de $15 \%$ (a) antes do RTFOT e (b) depois de RTFOT.
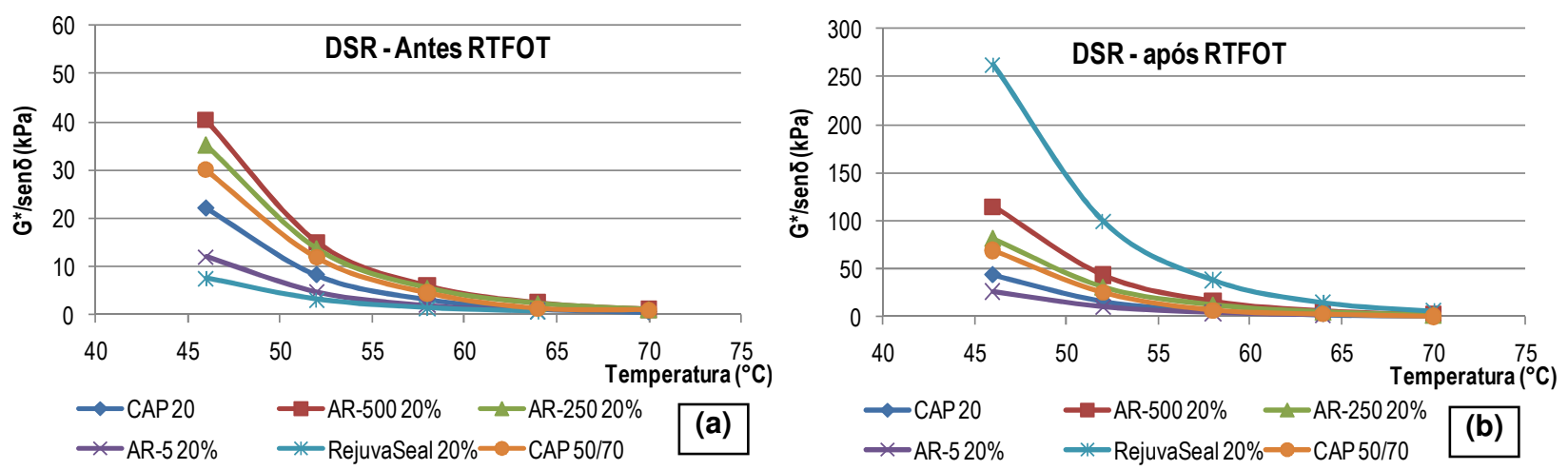

Figura 3. (a) G*/sen $\delta$ dos ligantes nos teores de adição de $20 \%$ (a) antes do RTFOT e (b) depois de RTFOT. 

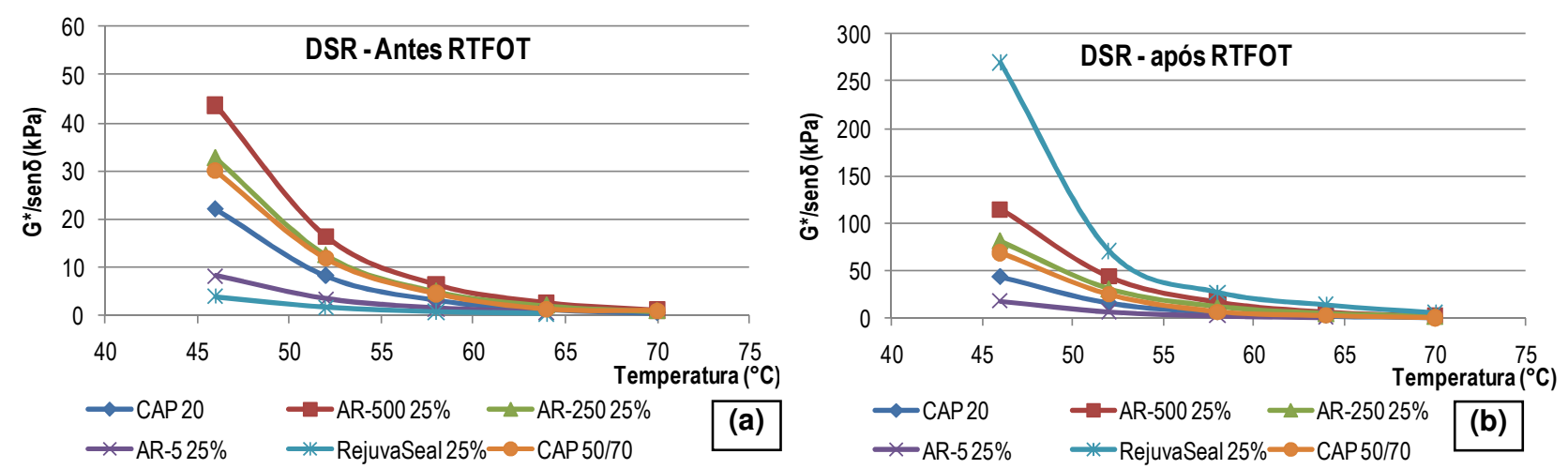

Figura 4. (a) G*/sen $\delta$ dos ligantes nos teores de adição de $20 \%$ (a) antes do RTFOT e (b) depois de RTFOT.

Segundo a especificação SUPERPAVE, os valores de $\mathrm{G}^{*} / \operatorname{sen} \delta$ devem ser maiores que $1,0 \mathrm{kPa}$ para o ligante em estado natural e superior a 2,2 $\mathrm{kPa}$ para o ligante que tenha sido submetido ao envelhecimento em curto prazo (RTFOT), sendo que as amostras com altos valores de $\mathrm{G}^{*} \mathrm{e}$ baixos valores de $\delta$ podem estar menos sujeitas a problemas de deformações permanentes de caráter viscoso, ou seja, maior resistência à deformação plástica. Os valores mínimos especificados foram alcançados para todas as configurações estudadas, com exceção das configurações com adição de RejuvaSeal. Aqui foi avaliado o comportamento do material submetido até a temperatura de $70^{\circ} \mathrm{C}$, que mostrou que os valores ficaram muito próximos ao limite mínimo especificado, ou seja, provavelmente se os ligantes e suas configurações fossem submetidos a temperaturas maiores que $70^{\circ} \mathrm{C}$, apresentaram compartimento reológico inferior ao mínimo especificado, trazendo prejuízos com relação à resistência à deformação plástica, reduzindo então sua vida útil.

Nota-se que após serem submetidos ao ensaio de RTFOT, os ligantes tiveram seu comportamento reológico alterado, principalmente aqueles ligantes que se apresentavam menos viscosos. Estes por sua vez, perderam frações leves, aumentando sua consistência, tendo um aumento significativo nos valores $\mathrm{de}^{*} / \operatorname{sen} \delta$. 0 ligante que mais sofreu alteração nesta característica foi o ligante com adição de RejuvaSeal, que em todas as porcentagens passou de uma condição de menor rigidez (antes RTFOT) para a maior rigidez após ser submetido ao envelhecimento em curto prazo.

Também foram avaliados resultados de ângulo de fase $(\delta)$, apresentados na Tabela 3.

Tabela 3 - Resultados dos ângulos de fase obtidos para os ligantes

\begin{tabular}{|c|c|c|c|c|c|c|}
\hline \multirow[b]{2}{*}{ Material } & \multicolumn{2}{|c|}{$15 \%$} & \multicolumn{2}{|c|}{$20 \%$} & \multicolumn{2}{|c|}{$25 \%$} \\
\hline & $\begin{array}{l}\text { Antes } \\
\text { RTFOT }\end{array}$ & $\begin{array}{c}\text { Após } \\
\text { RTFOT }\end{array}$ & $\begin{array}{l}\text { Antes } \\
\text { RTFOT }\end{array}$ & $\begin{array}{c}\text { Após } \\
\text { RTFOT }\end{array}$ & $\begin{array}{l}\text { Antes } \\
\text { RTFOT }\end{array}$ & $\begin{array}{c}\text { Após } \\
\text { RTFOT }\end{array}$ \\
\hline CAP velho & 84,7 & - & 84,7 & 82,0 & 84,7 & 82,0 \\
\hline CAP velho +RejuvaSeal & 83,1 & 67,3 & 83,3 & 65,1 & 84,2 & 65,6 \\
\hline CAP velho +AR-5 & 81,5 & 76,5 & 81,8 & 78,1 & 83,4 & 76,8 \\
\hline CAP velho +AR-250 & 80,2 & 73,6 & 80,6 & 73,7 & 81,4 & 73,9 \\
\hline CAP velho +AR-500 & 77,9 & 71,6 & 77,6 & 71,9 & 77,7 & 71,7 \\
\hline CAP $50-70$ & 79,1 & 77,3 & 79,1 & 77,3 & 79,1 & 77,3 \\
\hline
\end{tabular}

Dos resultados aqui apresentados se observa que os valores de ângulo de fase $(\delta)$ apresentaram redução após incorporação dos Agentes rejuvenescedores, o que comprova que as configurações testadas apresentariam, caso fossem utilizadas em campo, um melhor comportamento elástico. 
Após o ensaio de RTFOT houve uma inversão dos valores de ângulo de fase, ou seja, aqueles ligantes cuja viscosidade era mais baixa perderam frações leves, tornando o material mais consistente e, portanto mais viscoso, reduzindo então seu valor de ângulo de fase. Um bom exemplo deste fato é o ligante com adição de RejuvaSeal, que em todas as configurações passou de uma condição cujo ângulo de fase era o menor entre os demais, para a condição com maior valor de $\delta$.

\subsection{Análise química dos ligantes asfálticos}

Além da caracterização física, foram avaliados quais rejuvenescedores e quantidades fizeram com que o ligante extraído (envelhecido) apresentasse compostos químicos em quantidade semelhante à de um CAP 50-70. Essa análise teve como objetivo comparar as características químicas dos asfaltos rejuvenescidos com as do asfalto virgem tomado como padrão e verificar se o rejuvenescimento ocorreu de fato e se é possível encontrar um teor ótimo para cada rejuvenescedor ou até mesmo indicar o agente mais adequado.

\subsubsection{Composição SARA}

A cromatografia sobre coluna de sílica IATROSCAN permitiu separar diretamente os constituintes dos ligantes asfálticos avaliados nesta pesquisa. Foram obtidas as quatro famílias SARA, em função da polaridade do ligante, onde a partir do teor de cada fração foi possível verificar qual a quantidade e qual foi o rejuvenescedor que fez com que o ligante envelhecido se comportasse o mais próximo possível de um CAP 50-70 virgem.

A Tabela 4 traz os resultados obtidos para cada configuração estudada. Nela é possível observar os resultados obtidos para cada fração SARA em todas as configurações avaliadas, comparando-as inicialmente com os valores típicos estabelecidos pelo manual da Shell (Shell, 2003), onde a composição de Saturados deve estar entre 5 a 20\%; Aromáticos (40 a 65\%); Resinas (\% sem valor definido); Asfaltenos ( 5 a 25\%) e também com os valores obtidos para o CAP 50-70.

Tabela 4 - Composição química dos ligantes avaliados pelo método SARA

\begin{tabular}{lccccc}
\hline Ligantes & Saturados (5 a 20\%) & Aromáticos (40 a 65\%) & Resinas \% & Asfaltenos (5 a 25\%) & (Ic) \\
\hline CAP 50-70 & 7,9 & 44 & 33 & 16 & 0,31 \\
CAP velho & 8,8 & 34 & 36 & 22 & 0,44 \\
CAPvelho + 15\% AR 5 & 7,5 & 37 & 33 & 22 & 0,42 \\
CAPvelho + 20\% AR 5 & 8,3 & 40 & 35 & 32 & 0,34 \\
CAPvelho + 25\% AR 5 & 7,6 & 44 & 33 & 16 & 0,31 \\
CAPvelho + 15\% AR 250 & 7,5 & 37 & 34 & 22 & 0,42 \\
CAPvelho + 20\% AR 250 & 8,4 & 39 & 42 & 20 & 0,36 \\
CAPvelho + 25\% AR 250 & 7,5 & 30 & 35 & 24 & 0,38 \\
CAPvelho + 15\% AR500 & 6,8 & 34 & 35 & 24 & 0,45 \\
CAPvelho + 20\% AR 500 & 7,2 & 34 & 41 & 21 & 0,45 \\
CAPvelho + 25\% AR 500 & 8,2 & 29 & 39 & 23 & 0,42 \\
CAPvelho+15\% RejuvaSeal & 4,7 & 33 & 35 & 24 & 0,38 \\
CAPvelho+20\% RejuvaSeal & 6,3 & 34 & 35 & 22 & 0,44 \\
CAPvelho+25\% RejuvaSeal & 6,5 & 36 & & & 0,40 \\
\hline
\end{tabular}

*Ic = índice de instabilidade coloidal

É possível observar que o teor de asfaltenos não apresentou variação fora dos limites estabelecidos pela Shell (Shell, 2003). Entretanto, os teores de aromáticos por vezes apresentaram valores incompatíveis com aqueles tomados como padrão. Nota-se pela Tabela 4, que as únicas 
composições que se enquadraram nos padrões estabelecidos foram, respectivamente, o CAP+20\% de AR5 e CAP+25\% de AR5, recompondo principalmente a fração de aromáticos e redução das resinas e asfaltenos, produzindo uma ligante semelhante ao CAP 50-70.

Estes compostos aromáticos sofrem com a ação da temperatura e exposição ao ar, fazendo com que sejam condensados (reduzidos), produzindo resinas e parte das resinas produz asfaltenos, formando assim uma mistura bastante instável (Silva, 2005).

o Índice de Instabilidade Coloidal (Ic) de todos os ligantes avaliados pode ser enquadrado numa condição intermediária $(0,1 \leq \mathrm{Ic} \leq 0,5)$, onde quanto maior for Ic, maior será a Ic do asfalto e mais difícil a incorporação de um modificador, como por exemplo, um polímero (Glita, 1988). Entretanto, é possível notar que os ligantes CAP+20\% de AR5 e CAP+25\% de AR5, cujos compostos aromáticos enquadraram-se nas especificações da Shell (Shell, 2003), foram os que tiveram melhor Ic, ou seja, possuem os menores valores e, portanto, podem ser considerados os mais estáveis.

\subsubsection{Espectros RMN}

Os espectros de RMN 1H e RMN 13C fundamentaram a observação de qual ligante mais se aproximava de um ligante virgem, que para esta pesquisa foi o CAP 50-70. Observou-se que todas as configurações avaliadas apresentavam valores dos grupos Saturados e Aromáticos semelhantes aos de um CAP 50-70 tomado como padrão de referência. Entretanto, foram realizadas Análises de Variâncias (ANOVA) combinando pares de ligantes, onde se pretende verificar o nível de significância, ou seja, o quanto os resultados de um ligante rejuvenescido se aproximam dos resultados do CAP 50-70, ao se compararem os grupos de compostos Aromáticos e Saturados.

0 resumo dos resultados é apresentado na Tabela 5, informando o nível observado de significância "p", obtido na análise para cada um dos pares encontrados.

Tabela 5 - Níveis de Significância ( $p \%$ ) de valores obtidos pelo método RMN

\begin{tabular}{llll}
\hline Ligantes avaliados & (p\%) & Ligantes avaliados & (p\%) \\
\hline CAPvelho + 15\% AR 5 & $99,71 \%$ & CAPvelho + 15\% AR500 & $98,80 \%$ \\
CAPvelho + 20\% AR 5 & $99,80 \%$ & CAPvelho + 20\% AR 500 & $98,80 \%$ \\
CAPvelho + 25\% AR 5 & $99,50 \%$ & CAPvelho + 25\% AR 500 & $98,76 \%$ \\
CAPvelho + 15\% AR 250 & $99,00 \%$ & CAPvelho + 15\% RejuvaSeal & $97,90 \%$ \\
CAPvelho + 20\% AR 250 & $99,40 \%$ & CAPvelho + 20\% RejuvaSeal & $96,90 \%$ \\
CAPvelho + 25\% AR 250 & $99,95 \%$ & CAPvelho + 25\% RejuvaSeal & $94,30 \%$ \\
\hline
\end{tabular}

Quando comparados, todos os ligantes se aproximaram do CAP 50-70, sendo as seguintes as três melhores combinações em ordem decrescente de probabilidade: CAPvelho+25\% AR250 ( $p=99,95 \%)$, CAPvelho+20\% AR5 ( $p=99,8 \%)$ e por fim o CAPvelho+15\% AR5 (p=99,71\%) Entretanto, a escolha entre um dos três ligantes deverá ser baseada naquele que apresentou componentes da fração SARA compatíveis com o CAP 50-70, ou seja, CAPvelho+20\% AR5, uma vez que o ensaio de RMN só separa grupos de compostos aromáticos e saturados.

\section{CONCLUSÕES}

Observa-se que todas as porcentagens de incorporações de agentes rejuvenescedores ao CAP envelhecido favorecem a diminuição da consistência do ligante envelhecido, conferindo maiores valores de penetração e redução do ponto de amolecimento. Os ensaios realizados pela 
metodologia Superpave indicaram que a perda de massa pelo método RTFOT indicou que os valores permaneceram dentro dos limites estabelecidos pelas normativas brasileiras.

As misturas com RejuvaSeal por sua vez, não apresentaram bons resultados, pois o material apresenta baixa consistência, devido a possuir Destilado de Petróleo (32-42\%) e Rejuvenescedor 15-40\%) (Mah, 2008), e provavelmente o teor acrescido ao ligante envelhecido foi demasiadamente elevado, fazendo com que o ponto de amolecimento ficasse muito baixo e a penetração muito alta.

Todas as configurações de ligantes rejuvenescidos apresentaram valores obtidos de $\mathrm{G}^{*} / \mathrm{sen} \delta$ compatíveis o CAP 50-70, tomado como padrão de referência. Estes resultados também indicaram que os ligantes com os maiores valores de rigidez $\left(\mathrm{G}^{*} / \operatorname{sen} \delta\right)$, são mais resistentes ao acúmulo de deformação permanente, o que de fato já era esperado.

A avaliação química realizada por meio da cromatografia IATROSCAN permitiu separar e quantificar as quatro famílias SARA, onde foi possível verificar qual a quantidade e o rejuvenescedor que levou o ligante envelhecido a comportar-se mais próximo de um CAP 50-70, tomado como padrão de obras rodoviárias, principalmente em âmbito federal.

As composições que melhor se enquadraram nos padrões estabelecidos pela Shell (2003) foram, respectivamente, a composição com adição de $20 \%$ e $25 \%$ de AR-5, as quais recompuseram principalmente a fração de aromáticos com redução das resinas e asfaltenos, produzindo um ligante com frações SARA semelhantes ao CAP 50-70. Estes mesmos ligantes foram os que tiveram o melhor Índice de Instabilidade Coloidal (Ic), ou seja, possuem os menores valores sendo, portanto, considerados os mais estáveis dentre todas as configurações avaliadas.

Os espectros de RMN indicaram a adição de 25\% de AR-250 como a melhor opção para restabelecer as características químicas do ligante envelhecido, seguido da configuração com 15\% e 20\% de AR-5. Entretanto, como esta análise considera somente grupos de compostos aromáticos e saturados, a escolha do ligante em função da característica química deve ser realizada com base na cromatografia IATROSCAN, capaz de identificar todas as famílias SARA, ou seja, o melhor material seria aquele com a adição de $20 \%$ de AR-5.

\section{AGRADECIMENTOS}

Os autores agradecem à Universidade de Brasília e ao CNPq, pela oportunidade a aporte tecnológico para desenvolvimento desta pesquisa.

\section{REFERÊNCIAS}

AMERICAN ASSOCIATION OF STATE HIGHWAY AND TRANSPORTATION OFFICIALS. AASHTO M 332: Standard Specification for Performance-Graded Asphalt Binder Using Multiple Stress Creep Recovery (MSCR) Test. Washington, D.C., 2014.

AMERICAN SOCIETY FOR TESTING AND MATERIALS. ASTM D 4402. Standard Practice for Recovery of Asphalt from Solution Using the Rotary Evaporator. American Society for Testing and Materials. 1995.

Bonfim, V. \& Domingues, F. A. A. “Utilização de Fresagem e Reciclagem “In Situ” a Frio. Alternativas na Recuperação de Pavimentos Asfálticos". In: 29a Reunião Anual de Pavimentação, Volume 3, Cuiabá, MT, 1995.

CNT. Pesquisa rodoviária 2018: relatório gerencial. Brasília: In: Confederação Nacional do Transporte, 2018.

Corbett, L.W (1969). Composition of asphalt based on generic fractionation using solvent deasphalting, elution - adsorption chromatography and densimetric characterization. In. Anais. Chem., v.41, p.576. 1969. DOI:10.1021/ac60273a004

DNC. Portaria DNC nơ44. Departamento Nacional de Combustíveis, 1997.

Gaestel, C.; R. Shadja, e K.A. Lamminan. Contribution à la connaissance des proprietés dês bitumes routiers. Bull. Laboratoire des Ponts e Chausées. v.466, p.85-97.1971.

Fwa, T. F. Evaluation of Engineering Benefits of RJSeal Report preparated for Kalvani International Pte Ltd. Departmet of Civil Engineering. National University of Singapore, 2006.

Glita S. Contribuition à l'étude physico-chimie dês bitumes. Havre. Thèse (Doctorat em Sciences) - Faculté des Sciences et Techniques de l’Université du Havre.1988. 
IBGE. Estimativas populacionais para os municípios e para as Unidades da Federação brasileiros em 01.07.2017. Disponível em www.ibge.gov.br. Acesso em 01 outubro de 2017.

Jennings, P. W. e J.A. Pribanic. Binder Characterization and Evaluation by Nuclear Magnetic Resonance Spectroscopy. Final Report Strategic Highway Research Program SHRP-A-335, p. 150.1991.

Lima, A.T. Caracterização Mecânica de Misturas Asfálticas Recicladas a Quente. Dissertação de mestrado em Engenharia de Transportes. Programa de Mestrado em Engenharia de em Engenharia de Transportes, Centro de Tecnologia, Universidade Federal do Ceará, Fortaleza, 2003.

Mah, V. (2008) Concept of P4 - Perpetual Pavement Presentation Process. PRI- Pavement Rejuvenation International. Disponível em <http://www.rejuvaseal.com.hk/news/wp-content/uploads/2008/01/concept-of-p4-perpetual-pavimentpresentation-process.pdf $>$. Acesso em 07/03/2008.

Rejuvaseal (2010). Composition of "Rejuvaseal". Technical Specifications for Application of RejuvaSeal. Disponível em <http://www.rejuvaseal.com.hk/news/wp-content/uploads/2010/01/technical-standard-of-rejuvaseal-in-china.pdf>. Acesso em 14/02/2010.

Shakirullah, M.; I. Ahmad; H. Rahman; M.A. Khan.; M. Ishaq e A.A. Shah (2006). Spectral Analysis of Some Distillates From Asphalt Cracking and Evaluation of UTIMAC as Cracking Catalyst. Journal of the Chinese Chemical Society, vol. 53, n.1, p. 10591066. DOI: $10.1002 /$ jccs.200600141

Shell. SHELL Bitumen Handbook. SHELL, Inglaterra.2003.DOI: 10.1680/tsbh.58378.001

Silva, L. S. Contribuição ao estudo do envelhecimento de ligantes asfálticos. Influência na adição de polímeros e comportamento frente a radiação UV. Tese de Doutorado em Engenharia. UFRGS, Porto Alegre, 2005.

Silva, J.P.S.; M.M, Farias e A.M.G, Jiménez. "Avaliação do Efeito Selante de Agente Rejuvenescedor em Misturas Asfálticas”. In: $20^{\circ}$ Encontro de Asfalto, IBP Instituto Brasileiro de Petróleo, Gás e Biocombustíveis, Rio de Janeiro, Brasil. Maio de 2010.

Silva, J.P.S. Avaliação dos efeitos de produtos rejuvenescedores em misturas asfálticas. Tese de Doutorado. Programa de Pósgraduação em Geotecnia. Universidade de Brasília. 2011.

Silva, J.P.S e M.M. Farias. (2018). Avaliação química e ambiental de misturas asfálticas rejuvenescidas. Revista Matéria - UFRJ. v.23 n. 03, p.1-15. DOI: doi.org/10.1590/s1517-707620180003.0523

Zaumanis, M; R. B. Mallick e Frank, R. (2014). 100\% recycled hot mix asphalt: A review and analysis. Resources, Conservation and Recycling, v. 92, p. 230-245, 2016. DOI: 10.1016/j.resconrec.2014.07.007.

Zubaran, M. Avaliação do comportamento de misturas asfálticas recicladas mornas em laboratório e usina de asfalto. 2014. 151p. Dissertação (Mestrado em Ciências em Engenharia Civil) - Universidade Federal do Rio de Janeiro, Rio de Janeiro, 2014. 